\section{Drop re-review for big grant holders}

The fundamental concern with a second layer of review for holders of more than US $\$ 1$ million in grants from the US National Institutes of Health is that it takes us further from a meritocracy in which the best and highestimpact science is supported (Nature 489, 203; 2012).

Elite grant recipients have gone through reviewers' scrutiny and proved their productivity many times. Reviewers and grant administrators already check for potential funding overlap, one of the issues the reforms are designed to address. Peer review may be imperfect, but it is done by the scientific leaders in the relevant field, and there is no reason to believe that the judgement of an advisory council is superior.

The reluctance of most funding agencies to pay for research infrastructure necessitates either leveraging economies of scale from multiple grants or belonging to an academic institution that is wealthy enough to share the costs - a position not all researchers enjoy. The new policy would mainly affect the most productive principal investigators with multiple R01-type (individual project application) grants, widely considered to yield the most innovative research.

Taxpayers have the right to make the scientific community accountable for improving health, but this extra scrutiny may compromise attainment of that goal.

Hemant K. Roy Northwestern University, Evanston, Illinois, USA. h-roy@northwestern.edu

\section{EU scientific visa is a success in Ireland}

From experience in Ireland, I contend that Europe's scientific visa and the EURAXESS services can solve most of the problems regarding entry of foreign scientists into European research institutions and companies (Nature 488, 557; 2012). Fast-track immigration is an important consideration for internationally mobile scientists and helps to attract the best global talent to Europe.

Ireland, like the United Kingdom and Denmark, was not obliged to introduce the scientific visa under the 2005 European Union (EU) directive, but it was recognized that the visa would help to attract non-EU researchers to the country. The scheme, which offers a free and fast service, has been successfully operating in Ireland since 2007 and is open to universities and companies.

Visas are issued rapidly and work permits are not required. Researchers' families can accompany them immediately and use public schooling. Family members have access to the job market and can stay on to look for a job after the contract ends.

More than 200 EURAXESS

offices in 40 European countries, including Ireland, help foreign researchers to move across Europe by providing practical information. The EURAXESS portal (http://ec.europa.eu/ euraxess) is a free advertising forum for EU research positions.

\section{Conor O'Carroll Irish}

Universities Association, Dublin, Ireland.conor.ocarroll@iua.ie

\section{Who discovered Universe expansion?}

Controversy persists over who first found that the Universe is expanding. Last year, Mario Livio quashed suggestions that Georges Lemaître's 1927 theoretical prediction of expansion was deliberately suppressed (Nature 479, 171$173 ; 2011)$. Since then, another contender has emerged.

The joint NASA and Infrared
Processing and Analysis Center Extragalactic Database of Galaxy Distances, in Pasadena, California, which I co-lead, has tabulated and made public the historical distance estimates published by Edwin Hubble and his contemporaries to prove expansion (see I. Steer J. R. Astron. Soc. Can. 105, 18-20; 2011). These reveal that measurements by a Swedish astronomer, Knut Lundmark, were much more advanced than formerly appreciated.

Lundmark was the first person to find observational evidence for expansion, in 1924 - three years before Lemaitre and five years before Hubble. Lundmark's extragalactic distance estimates were far more accurate than Hubble's, consistent with an expansion rate (Hubble constant) that was within $1 \%$ of the best measurements today.

However, Lundmark's research was not adopted because it relied on one unproven method (galaxy diameters), cross-checked with one unproven distance to the Andromeda galaxy, which was derived from a type Ia supernova observed in 1885 and mistaken for a normal nova (W. Huggins and W. F. Denning Nature 32, 465-466; 1885).

Hubble's research in 1929 yielded a value for the Hubble constant that was inaccurate by almost an order of magnitude. It was adopted because it was derived from multiple methods - including one still in use (brightest stars) - and was cross-checked with multiple galaxies with distances based on proven Cepheid star variables.

Lundmark established observational evidence that the Universe is expanding. Lemaître established theoretical evidence. Hubble established observational proof.

Ian Steer NASA/IPAC Extragalactic Database of Galaxy Distances, Pasadena, California, USA. ian@colosseum.com

\section{Environment in Queensland at risk}

In the state of Queensland, Australia, hard-won environmental protections are under threat.

In April this year, Queensland elected a new government that is prodevelopment and pro-mining. These activities have been burgeoning over the past few years, prompting the United Nations Educational, Scientific and Cultural Organization (UNESCO) to investigate whether the Great Barrier Reef should be included on the List of World Heritage in Danger (see http://whc.unesco.org/en/ documents/117104).

The government is also moving to dismantle the Wild Rivers Act, which was designed to protect and manage Queensland's last remaining pristine rivers and catchments in Cape York. These rivers have the richest diversity of freshwater fish in Australia, including the sawfish Pristis microdon and the speartooth shark Glyphis glyphis. These are considered by the International Union for Conservation of Nature to be critically endangered and endangered, respectively. Australia is one of the last countries in the world where populations of these fish are viable.

Ministers have nevertheless declared that a regional plan for Cape York will "unashamedly" fast-track development and "absolutely" secure a place for mining in these areas (see go.nature.com/e6hbzc).

These threats to Australia's aquatic environment are being compounded by government cuts to staff in environmental and fisheries management across public institutions. Andrew Chin, Jimmy White James Cook University, Townsville, Australia. andrew.chin@jcu.edu.au Nick Dulvy Simon Fraser University, Burnaby, Canada. 